\title{
Renoprotective effects of ursolic acid on ischemia/reperfusion-induced acute kidney injury through oxidative stress, inflammation and the inhibition of STAT3 and NF- $\kappa$ B activities
}

\author{
JUN PENG, XINGFENG REN, TIANBIAO LAN, YAN CHEN, ZIYUN SHAO and CHENG YANG \\ Department of Nephrology, Wuhan General Hospital of Guangzhou Military Area, \\ Wuhan, Hubei 430030, P.R. China
}

Received April 8, 2015; Accepted June 22, 2016

DOI: $10.3892 / \mathrm{mmr} .2016 .5654$

\begin{abstract}
Ursolic acid, a pentacyclic triterpene compound with low toxicity and easy availability, has a variety of biological activities, including antitumor, antioxidant, antihepatitis, anti-inflammatory and antibacterial effects. The present study aimed to investigate the renoprotective effects of ursolic acid on ischemia/reperfusion-induced acute kidney injury (I/R-IAKI) in rats associated with its antioxidant and anti-inflammatory effects, as well as interference with the signal transducer and activator of transcription (STAT)3/nuclear factor (NF)- $\mathrm{kB}$ signaling pathway. The present study demonstrated that pre-treatment with ursolic acid significantly increased renal functioning and attenuated increases of serum angiotensin II levels in rats subjected to I/R-IAKI. In addition, I/R-IAKI-induced inflammation and oxidative stress were significantly reduced by pre-treatment with ursolic acid. Furthermore, ursolic acid significantly suppressed the upregulation of STAT3, NF- $\mathrm{KB}$ and caspase- 3 activities in rats following I/R-IAKI. These results indicated that ursolic acid may be a potential drug for reducing I/R-IAKI through suppression of inflammation and oxidative stress damage, as well as modulation of STAT3 and NF- $\mathrm{KB}$ activities.
\end{abstract}

\section{Introduction}

Acute kidney injury (AKI), referred to acute renal failure, is a type of clinical syndrome caused by multiple factors. Different clinical manifestations occur and the predominant characteristic is a decrease in the rate of glomerular filtration (1). AKI

Correspondence to: Mr. Xingfeng Ren, Department of Nephrology, Wuhan General Hospital of Guangzhou Military Area, 627 Wu Luo Road, Wuhan, Hubei 430030, P.R. China E-mail: pppjunpeng@163.com

Key words: ursolic acid, ischemia/reperfusion-induced acute kidney injury, inflammation, oxidative stress, signal transducer and activator of transcription 3 , nuclear factor- $\mathrm{kB}$ is a common clinical critical disease, with incidence rates as high as $2 / 3$ in AKI disease. Three major parallel complications can occur, including respiratory failure and shock, in AKI patients, and these are independent risk factors to predict the mortality of patients with AKI. According to a previous report, the incidence of AKI in hospitals was $4.05 \%$ and the mortality rate was as high as $12.7 \%$ (2). In China's comprehensive hospitals, patients with AKI accounted for 3.19\% of hospitalized patients (3). In the USA in 2011, AKI medical costs were as much as $\$ 4.7$ billion (4). Although modern medical treatment conditions and medical technology have improved, and a variety of alternative treatments exist, AKI morbidity and mortality remains high. This is a difficult problem worldwide (5).

The mechanism of ischemia/reperfusion-induced acute kidney injury (I/R-IAKI) remains to be fully elucidated. It is known that at least three factors are involved: Reactive oxygen species, neutrophils and the Complement system (6). Previous research has demonstrated that I/R-IAKI is closely associated with the generation of reactive oxygen free radicals (7). It was also confirmed that oxygen free radicals in the I/R-IAKI serve a highly important role (8). I/R-IAKI generates oxygen free radicals, which have strong oxidation activity, and can damage biological molecules, particularly lipids (9). Cell membrane and lipid peroxidation of unsaturated fatty acids on mitochondrial and lysosomal membranes, can lead to damage to the structure and function of renal tubular cells (9). Eventually lipid peroxide breaks down into malondialdehyde (MDA), and MDA also serves a cytotoxic effect, adding to I/R injury.

Ursolic acid is widely distributed in nature and exists in numerous plants, including hawthorn, bearberry, Chinese elder herb, fructus ligustri lucidi, lanatoside kiwi fruit, dogwood, wild rose fruit, Chinese sage, gentiana, Prunella vulgaris, spreading hedyotis herb, chrysanthemum cuckoo, azalea and forsythia (10). Ursolic acid is a pentacyclic triterpene compound, with the molecular formula for $\mathrm{C}_{30} \mathrm{H}_{48} \mathrm{O}_{3}$ and a molecular weight of 456.68 (Fig. 1). Certain Chinese herbal medicines containing ursolic acid exert a variety of effects, including effects on blood stasis, as an analgesic, as an anti-inflammatory, and as a protective agent in the liver (11). Modern pharmacological studies have found that ursolic acid 
has extensive biological effects, particularly in antitumor, antioxidant, resistance to viral hepatitis and anti-inflammatory (12). The present study hypothesized that ursolic acid may affect I/R-IAKI, thus functioning in antioxidant, anti-inflammatory and the STAT3/NF- $\mathrm{B}$ signaling pathway. The present study aimed to demonstrate the renoprotective effects of ursolic acid on I/R-IAKI through oxidative stress, inflammation and inhibiting STAT3 and NF- $\kappa \mathrm{B}$ activities in rat.

\section{Materials and methods}

Reagents. Ursolic acid (purity 90\%) and sodium pentobarbital were obtained from Sigma Chemical Co. (St. Louis, MO, USA). Angiotensin-II kits were obtained from SpiBio (Bertin-Pharma, Lille Cedex, France). Tumor necrosis factor (TNF) $\alpha$, interleukin (IL)-6, IL- $1 \beta$ and nuclear factor (NF)- $\mathrm{B}$ p65 unit, microvessel density and superoxide dismutase (SOD) specific enzyme-linked immunosorbent assay (ELISA) kits were obtained from Jiancheng Bioengineering Institute (Nanjing, China).

Animals andI/R-IAKImodel.A total of 30 male Sprague-Dawley rats (age, 2-month-old; weight, 230-300 g), were purchased from the Animal Experiment Center of Wuhan University (Wuhan, China) maintained under a $12 \mathrm{~h}$ dark/light cycle at $23-24^{\circ} \mathrm{C}$ with a relative humidity of 40-60\%. All studies performed on animals were approved by the Institutional Animal Care and the local Ethics Committee. Sprague-Dawley rats were anaesthetized using sodium pentobarbital $(40 \mathrm{mg} / \mathrm{kg}$, Sigma Chemical Co.). A right nephrectomy was performed from a dorsal incision. Additionally, by means of dorsal incision, the left kidney and renal vessels were exposed and occluded with a vascular clip. After 45-90 min clamping, the vascular clip was removed and I/R-IAKI model rat was successful created.

Experimental design. The 30 rats were divided into three groups (n=10/group): i) Sham group with sham-operated rats administered with saline; ii I/R-IAKI group where the I/R-IAKI model rats were administered with saline; iii) ursolic acid group where the I/R-IAKI model rats were administered with $10 \mathrm{mg} / \mathrm{kg}$ ursolic acid twice daily for 4 days (13).

Blood pressure measurement. Sprague-Dawley rats were individually caged at $37^{\circ} \mathrm{C}$ for $30 \mathrm{~min}$ and were subsequently transferred to a fixed measurement cage following warming at $37^{\circ} \mathrm{C}$. Blood pressure was measured using a Narcosystem Device (North Dakota). The Sprague-Dawley rats were restrained and body length and weight were measured.

Renal functioning evaluation. Following anesthesia with 5\% chloral hydrate, blood samples were extracted from the rat tail vein into test tubes and centrifuged at 3,000 x $\mathrm{g}$ for $10 \mathrm{~min}$ at $4^{\circ} \mathrm{C}$. Following centrifugation, the supernatants were collected and the serum creatinine and cystatin-C levels were measured using a Roche/Hitachi 917 autoanalyzer (Roche Diagnostics, Indianapolis,. IN, USA), according to the manufacturer's protocol.

Measurement of angiotensin-II. Blood samples were extracted from the tail vein into test tubes and centrifuged at $3,000 \mathrm{xg}$

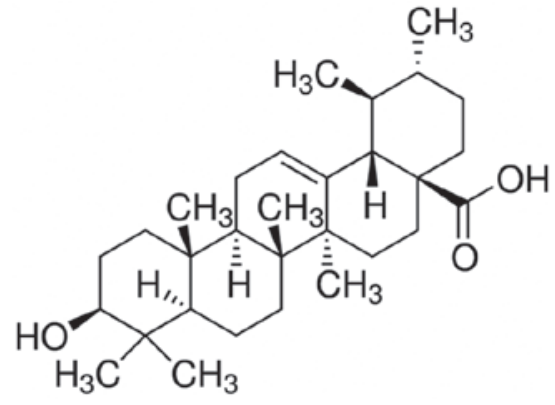

Figure 1. Chemical structure of ursolic acid.

for $10 \mathrm{~min}$ at $4^{\circ} \mathrm{C}$. Following centrifugation, the supernatants were collected and the levels of angiotensin-II were measured using a specific ELISA (SpiBio), according to the manufacturer's protocol.

Measurement of inflammatory status and oxidative stress. Blood samples were extracted from the tail vein into test tubes and centrifuged at $3,000 \mathrm{x}$ g for $10 \mathrm{~min}$ at $4^{\circ} \mathrm{C}$. Following centrifugation, the supernatants were collected and the levels of TNF- $\alpha$, IL-6, IL-1 $\beta$, IL-10, MDA, SOD and NF- $\kappa$ B p65 unit were measured using specific ELISA kits (Jiancheng Bioengineering Institute), according to the manufacturer's protocols.

Western blotting analysis of STAT3. The rats were sacrificed by brief halothane overdose and renal tissues samples were homogenated with radioimmunoprecipitation lysis buffer (Beyotime Institute of Biotechnology, Jiangsu, China). The solution was centrifuged at $3,000 \mathrm{x}$ g for $10 \mathrm{~min}$ at $4^{\circ} \mathrm{C}$. Following centrifugation, the supernatants were collected and the protein contents were measured using a Coomassie brilliant blue assay (Beyotime Institute of Biotechnology). Equivalent quantities $(40 \mu \mathrm{g})$ of protein were separated by electrophoresis using $12 \%$ sodium dodecyl sulfate-polyacrylamide gels and were subsequently transferred onto nitrocellulose filter membranes (EMD Millipore, Billerica, MA, USA). The membranes were blocked in 5\% non-fat milk for $1 \mathrm{~h}$ at $37^{\circ} \mathrm{C}$. Following blocking, the membranes were incubated with the corresponding primary antibodies against anti-STAT3 (1:2,000; cat. no. sc-8001) and anti- $\beta$-actin (cat. no. 130656; 1:5,000) both from Santa Cruz Biotechnology, Inc. (Santa Cruz, CA, USA) overnight at $4^{\circ} \mathrm{C}$. Following this, the membranes were washed with Tris-buffered saline with Tween 20 and incubated with goat anti-rabbit secondary antibodies (1:5,000; BestBio, Inc., Shanghai, China; cat. no. BB-2202-1) for 2-3 h at room temperature. The bands were visualized using enhanced chemiluminescence detection (Amersham, Arlington Heights, IL, USA).

Measurement of caspase-3. The rats were sacrificed by brief halothane overdose and renal tissues samples were homogenated with radioimmunoprecipitation lysis buffer (Beyotime Institute of Biotechnology, Jiangsu, China). The solution was centrifuged at $3,000 \mathrm{x} \mathrm{g}$ for $10 \mathrm{~min}$ at $4^{\circ} \mathrm{C}$. Following centrifugation, the supernatants were collected and the protein contents were measured using a Coomassie brilliant blue assay 
(Beyotime Institute of Biotechnology). Equivalent quantities of protein $(50 \mu \mathrm{g})$ were incubated with Ac-LEHD-pNA (Beyotime Institute of Biotechnology) at $37^{\circ} \mathrm{C}$ for $2 \mathrm{~h}$ in the dark and the caspase- 3 level was measured at an absorbance of $405 \mathrm{~nm}$.

Statistical analysis. The data are expressed as the mean \pm standard error of the mean. Statistical analyses were performed where appropriate with the Student-Newman-Keuls method using SPSS 17.0 (SPSS, Inc., Chicago, IL, USA). P<0.05 was considered to indicate a statistically significant difference.

\section{Results}

Ursolic acid demonstrated no effect on blood pressure monitoring. To assess the renoprotective effects of ursolic acid on I/R-IAKI, blood pressure monitoring was performed. As shown in Fig. 2, no significant changes were determined between the groups $(\mathrm{P}=0.911)$.

Ursolic acid exerted a renoprotective effect on renal functioning. The present study next probed the renoprotective effects of ursolic acid on I/R-IAKI renal functioning. I/R-IAKI significantly increased the levels of serum creatinine in rats compared with that of the sham group (Fig. 3; $\mathrm{P}=0.0001)$. However, I/R-IAKI revealed no effect on the serum levels of cystatin-C (Fig. 3). Additionally, the increased serum creatinine was effectively inhibited and the serum cystatin- $\mathrm{C}$ levels were effectively increased by treatment with ursolic acid, as compared with those of the I/R-IAKI group (Fig. 3; P=0.0009).

Ursolic acid decreased levels of angiotensin-II. To investigate the renoprotective effects of ursolic acid on I/R-IAKI, the serum angiotensin-II level was determined in the present study. I/R-IAKI increased the serum levels of angiotensin-II in the rats compared with that of the sham group (Fig. 4; $\mathrm{P}=0.0038$ ). Treatment with ursolic acid suppressed the enhancement of serum angiotensin-II levels in I/R-IAKI rats (Fig. 4; P=0.0042), when compared with the I/R-IAKI rats.

Ursolic acid reduced inflammation. To further determine the renoprotective effects of ursolic acid on I/R-IAKI, the serum levels of TNF- $\alpha$, IL- 6 , IL- $1 \beta$ and IL-10 were measured following injury. I/R-IAKI significantly increased the serum levels of TNF- $\alpha$, IL-6, IL-1 $\beta$ and IL-10 compared with the sham group (Fig. 5; $\mathrm{P}=0.0009, \mathrm{P}=0.0051, \mathrm{P}=0.0007$ and $\mathrm{P}=0.0076$, respectively). As presented in Fig. 5, pre-treatment with ursolic acid significantly reduced the I/R-IAKI-induced serum levels of TNF- $\alpha$, IL-6, IL-1 $\beta$ and IL-10 in the I/R-IAKI rats $(\mathrm{P}=0.0011, \mathrm{P}=0.0066, \mathrm{P}=0.0053$ and $\mathrm{P}=0.0072$, respectively).

Ursolic acid reduced oxidative stress. Additionally, the present study determined the serum levels of MDA and SOD following injury. As shown in Fig. 6, I/R-IAKI caused increased serum levels of MDA and suppressed the serum levels of SOD in rats $(\mathrm{P}=0.0039$ and $\mathrm{P}=0.0026$, respectively). Oxidative stress was effectively reduced following treatment ursolic acid (Fig. 6; $\mathrm{P}=0.0016$ and $\mathrm{P}=0.0079$, respectively).

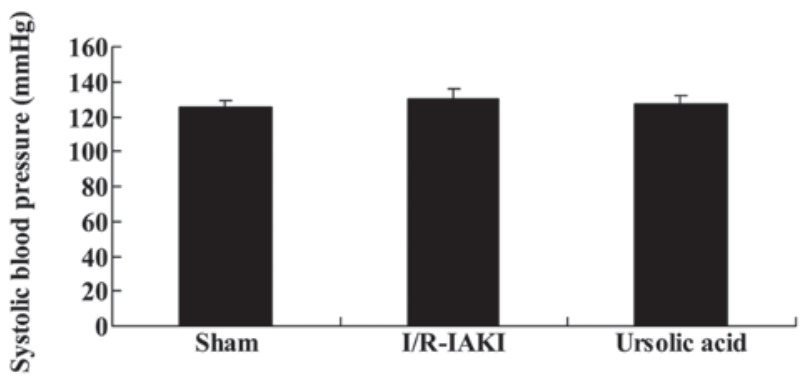

Figure 2. Effects of ursolic acid on systolic blood pressure in rats subjected to IR/IAKI. The data are expressed as the mean \pm standard error of the mean. IR/IAKI, ischemia/reperfusion-induced acute kidney injury.
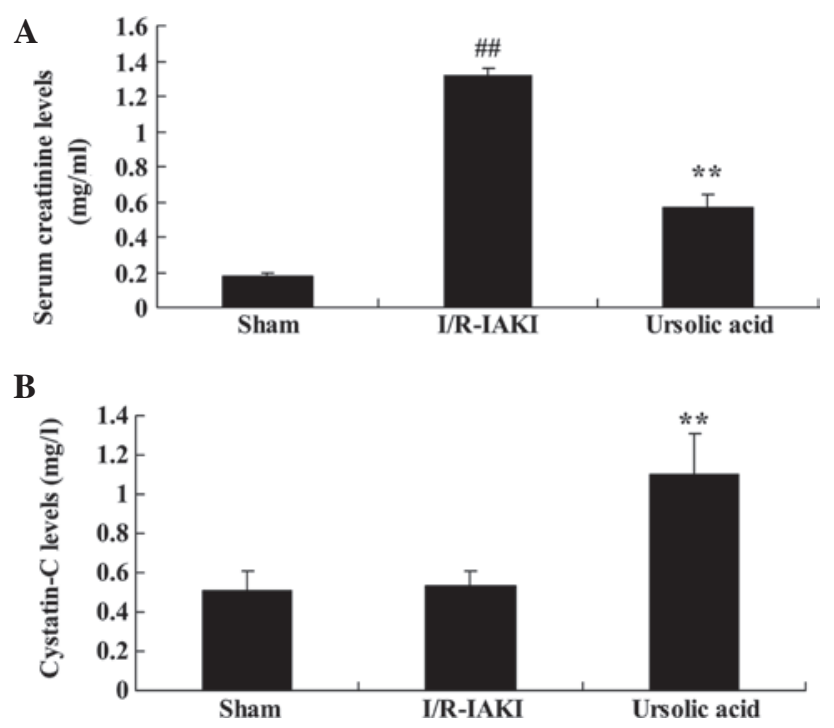

Figure 3. Effects of ursolic acid on renal functioning in rats subjected to I/R-IAKI. (A) Serum creatinine and (B) serum Cystatin-C levels. The data are expressed as the mean \pm standard error of the mean $\left({ }^{\# \#} \mathrm{P}<0.01\right.$ compared with sham group; ${ }^{* *} \mathrm{P}<0.01$ compared with I/R-IAKI group). IR/IAKI, ischemia/reperfusion-induced acute kidney injury.

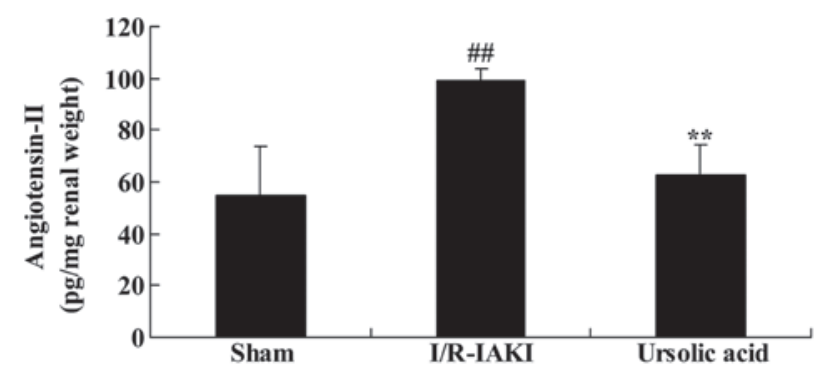

Figure 4. Effects of ursolic acid on angiotensin-II levels in the kidneys of rats subjected to I/R-IAKI. The data are expressed as the mean \pm standard error of the mean ${ }^{\# \#} \mathrm{P}<0.01$ compared with sham group; ${ }^{* *} \mathrm{P}<0.01$ compared with I/R-IAKI group). IR/IAKI, ischemia/reperfusion-induced acute kidney injury.

Ursolic acid reduced levels of STAT3. To further investigate the renoprotective effects of ursolic acid on I/R-IAKI, the protein expression levels of phosphorylated (p)-STAT3 were measured following injury. I/R-IAKI rats exhibited a significant progressive increase in the protein expression levels of p-STAT3 compared with that of the sham group (Fig. 7; 
A



C

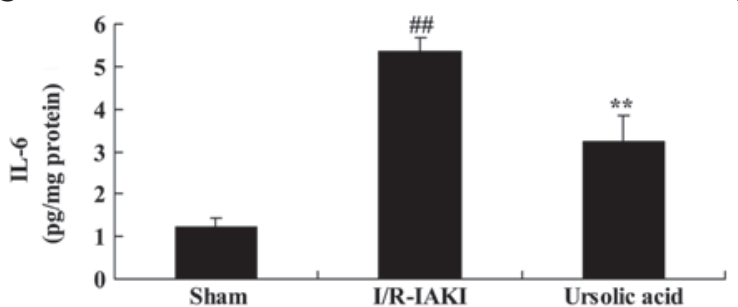

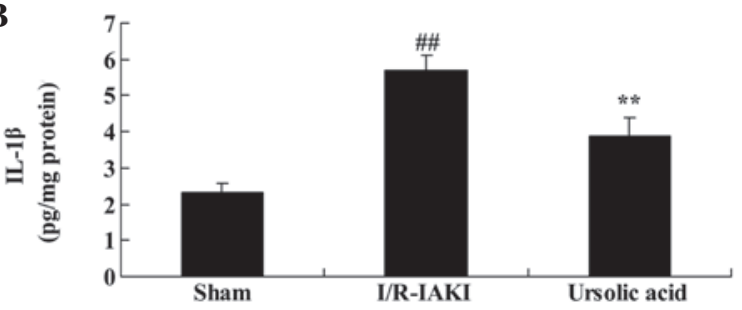

D

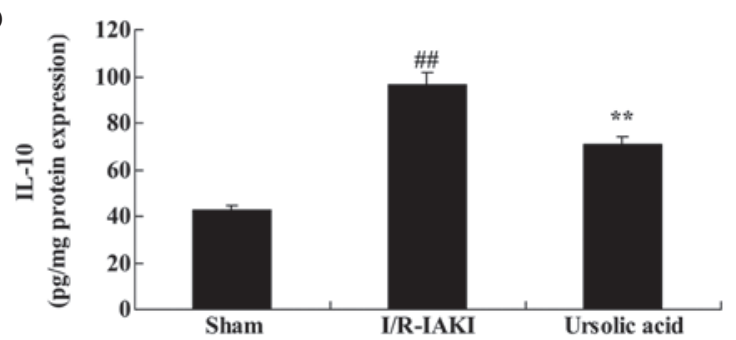

Figure 5. Effects of ursolic acid on inflammation in rats subjected to I/R-IAKI. Serum levels of (A) TNF- $\alpha$, (B) IL-1 $\beta$ (C) IL-6 and (D) IL-10. The data are expressed as the mean \pm standard error of the mean $\left({ }^{\# \#} \mathrm{P}<0.01\right.$ compared with sham group; ${ }^{* *} \mathrm{P}<0.01$ compared with I/R-IAKI group). IR/IAKI, ischemia/reperfusion-induced acute kidney injury; TNF, tumor necrosis factor; IL, interleukin.
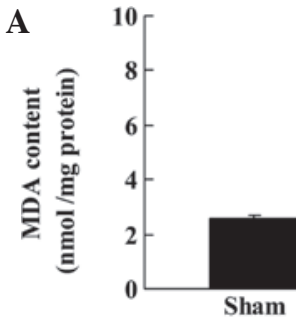

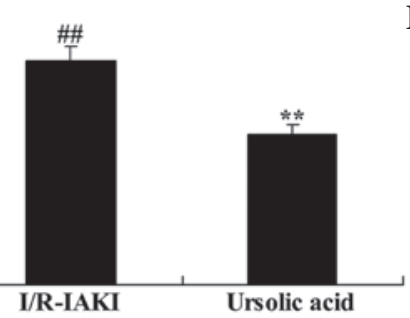

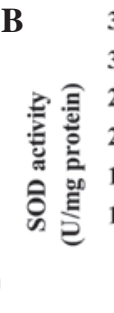

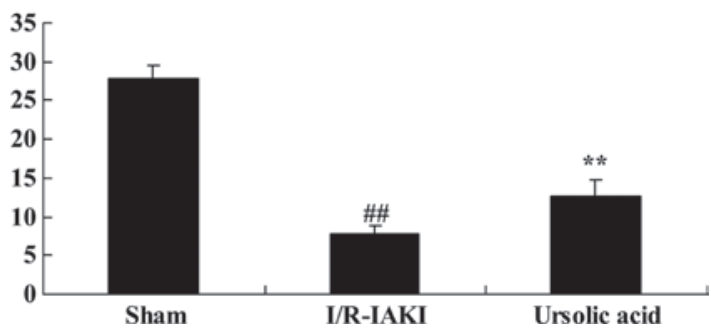

Figure 6. Effects of ursolic acid on oxidative stress in rats subjected to I/R-IAKI. Serum levels of (A) MDA and (B) SOD. Values are expressed as the mean \pm standard error of the mean. ${ }^{\# \#} \mathrm{P}<0.01$ compared with sham group; ${ }^{* *} \mathrm{P}<0.01$ compared with I/R-IAKI group. IR/IAKI, ischemia/reperfusion-induced acute kidney injury; SOD, superoxide dismutase; MDA, malondialdehyde.
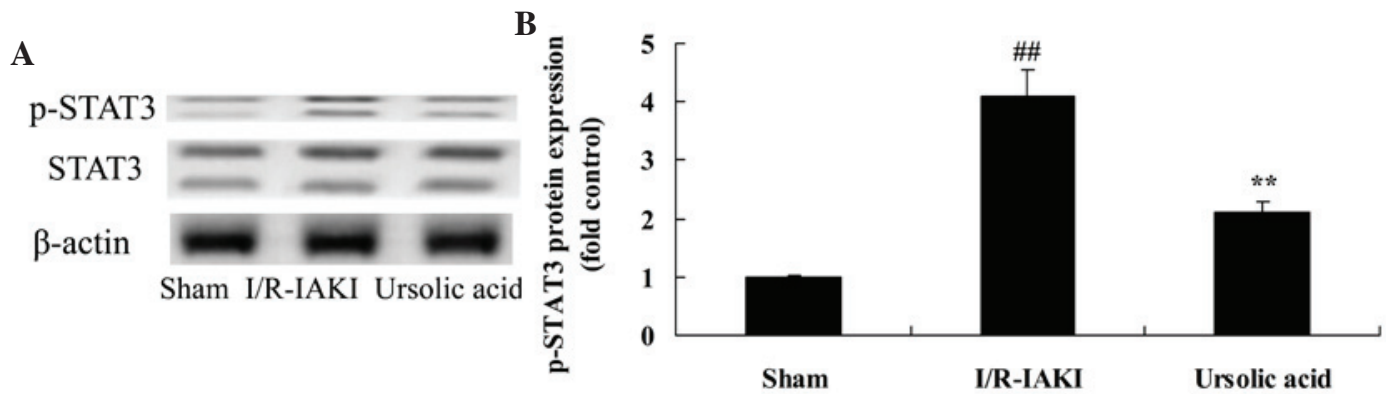

Figure 7. Effects of ursolic acid on the activation of STAT3 in the rats subjected to I/R-IAKI. (A) Representative western blotting of the protein expression of p-STAT3 and (B) quantified levels of p-STAT3 determined by densitometric analysis of band intensities. The data are expressed as the mean \pm standard error of the mean $\left({ }^{\# \#} \mathrm{P}<0.01\right.$ compared with sham group; ${ }^{* *} \mathrm{P}<0.01$ compared with I/R-IAKI group). IR/IAKI, ischemia/reperfusion-induced acute kidney injury; p-STAT, phosphorylated signal transducer and activator of transcription.

$\mathrm{P}=0.0011)$. Following ursolic acid treatment, the protein expression levels of p-STAT3 were significantly reduced in I/R-IAKI rats (Fig. 7; $\mathrm{P}=0.0044$ ).

Ursolic acid decreased $N F-\kappa B$ expression in the I/R-IAKI rats. The levels of NF- $\kappa \mathrm{B}$ were measured following injury. In the I/R-IAKI rats, NF- $\kappa \mathrm{B}$ level was significantly augmented compared with the rats of the sham group (Fig. 8; $\mathrm{P}=0.0016$ ). Following treatment with ursolic acid, the increased level of
$\mathrm{NF}-\kappa \mathrm{B}$ was significantly inhibited compared to that of the I/R-IAKI group (Fig. 8; P=0.0003).

Ursolic acid reduced the increase in caspase-3 observed in I/R-IAKI rats. To further understand the renoprotective effects of ursolic acid on I/R-IAKI, the caspase-3 activity was assessed following injury. As shown in Fig. 9, I/R-IAKI caused an increase in caspase-3 activity in rats compared with that of the sham group (Fig. 9; $\mathrm{P}=0.00032$ ). Following treatment with 


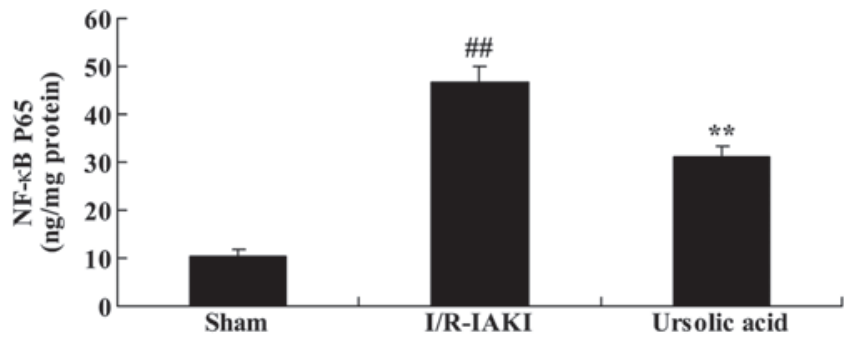

Figure 8. Effects of ursolic acid on the activation of NF- $\kappa \mathrm{B}$ p65 in rats subjected to I/R-IAKI. The data are expressed as the mean \pm standard error of the mean $\left({ }^{\# \#} \mathrm{P}<0.01\right.$ compared with sham group; ${ }^{* *} \mathrm{P}<0.01$ compared with I/R-IAKI group). NF- $\kappa \mathrm{B}$, nuclear factor- $\kappa \mathrm{B}$; IR/IAKI, ischemia/reperfusion-induced acute kidney injury.

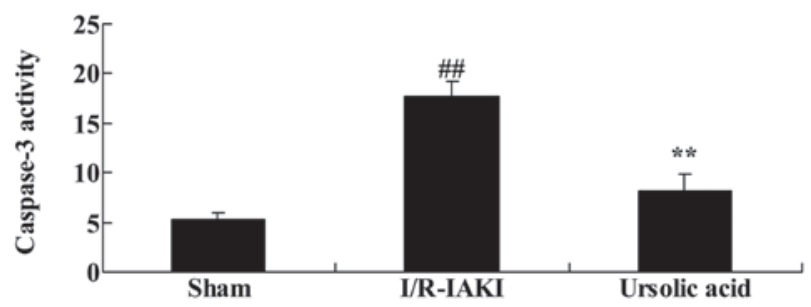

Figure 9. Effects of ursolic acid on caspase-3 activity in rats subjected to I/R-IAKI. The data are expressed as the mean \pm standard error of the mean $\left({ }^{\# \#} \mathrm{P}<0.01\right.$ compared with sham group; ${ }^{* *} \mathrm{P}<0.01$ compared with I/R-IAKI group). IR/IAKI, ischemia/reperfusion-induced acute kidney injury.

ursolic acid, the increase in caspase-3 activity was significantly weakened in the I/R-IAKI rats (Fig. 9; P=0.00071).

\section{Discussion}

At present, the underlying mechanisms of I/R-IAKI remains unclear. In the I/R-IAKI process, a large number of free radicals are generated, resulting in calcium overload and cell apoptosis. This interferes with energy metabolism. Neutrophil infiltration is important role in vascular endothelial injury (14). Existing literature reported that I/R-IAKI free radical oxygen outbreak is important for pathogenesis (15). In the present study, the results confirmed that pre-treatment with ursolic acid caused no affect on blood pressure monitoring and increased renal functioning in I/R-IAKI rat, resulting in a significant change that may decrease the I/R-IAKI-induced serum angiotensin-II level. Li et al (16) reported that ursolic acid protected the brain against ischemic injury in mice. Tundis et al (17) reported that the isolation of ursolic acid inhibits angiotensin-converting enzyme inhibitory activity (17). A number of results suggested that the protective effect of ursolic acid can be used for the treatment of I/R-IAKI.

The I/R-IAKI systemic response may be an initial cause of inflammation. Kidney I/R injury can lead to kidney synthesis of pro-inflammatory factors, including IL-6, IL-1 and TNF- $\alpha$. This can also quickly produce a variety of chemokines, including keratinocyte-derived chemokine, a rat chemotactic factor similar to that of the IL- 8 that can also be used as an I/R-IAKI biochemical marker (18). In the present experimental setting, ursolic acid treatment significantly reduced the I/R-IAKI-induced expression of TNF- $\alpha$, IL-6, IL-1 $\beta$ and
IL-10 in I/R-IAKI rats. Senthil et al (19) indicated that ursolic acids protects against isoproterenol-induced myocardial ischemia through antioxidant and anti-inflammatory functions in rats (19). This accumulation suggested that the anti-inflammatory effect of ursolic acid is associated with protection against I/R-IAKI.

Under normal physiological conditions, the body maintains normal metabolic processes and can produce the correct quantity of free radicals. A large number of free radicals has a damaging effect to the body. However, a large number of enzymes can remove free radicals from the body exerting antioxidant properties to prevent damage due to free radicals (20). The radical scavenging enzyme system, including SOD, MDA, hydrogen peroxide enzyme peptide, glutathione peroxidase and I/R-IAKI, cause cytotoxicity damage factors besides hypoxemia, can also directly induce inflammatory reactions in renal parenchymal cells resulting in ischemic acute renal failure (21). The present study quantified the serum levels of MDA and SOD, and these were significantly suppressed by treatment with ursolic acid in the I/R-IAKI rat. Ma et al (22) suggested that ursolic acid protects against $\mathrm{CCl}_{4}$-induced oxidative stress and inflammation of the liver in mice. Ramachandran et al (23) concluded that ursolic acid protects against exposure to ultraviolet $\mathrm{B}$-induced reactive oxygen species through modulation of antioxidants (23). The present results indicated that the protective effect of ursolic acid on I/R-IAKI is correlated with the modulation of oxidative stress.

JAKs (JAK1, JAK2, JAK3 and TYK2) and STATs protein family (STAT1, STAT2, STAT3, STAT4, STAT5a and STAT5b, STAT6) have been identified as signal transduction pathways in cells (24). Numerous cytokines, including IFN, IL-2, IL-4, IL-6 and CNTF, and growth factors, including EGF, PDGF and CSF, all using the signal transduction pathway induced cell proliferation, differentiation and apoptosis. These proteins may serve a special and pleiotropic biological function on immune regulation, hematopoietic stem cells, cancer, nervous system and embryonic development (25). Previous studies have shown that the JAK/STAT signal transduction pathways are involved in the development of the central nervous system and nerve cell proliferation, survival, differentiation, and they are closely associated with brain tumors, central nervous system diseases, including cerebral ischemia, and pathological physiological processes (26). The present study found that ursolic acid treatment reduced the increase of the protein expression levels of p-STAT3 in I/R-IAKI rats. Pathak et al (27) suggested that ursolic acid suppresses the cell proliferation of multiple myeloma cells and inhibits STAT3 activation (27). Ma et al (28) suggested that ursolic acid protects against oxidative DNA damage and inflammation by inhibiting the STAT3 and NF- $\kappa$ B activities (28). Taken collectively, ursolic acid may protect against I/R-IAKI-induced oxidative damage and inflammation by inhibiting STAT3.

It was previously shown in animal models that hypoxemia can lead to the rapid activation of the NF- $\kappa \mathrm{B}$ signaling system, causing the transcription and synthesis of pro-inflammatory factors (29). It was also shown that in the stage of the ischemia, the NF- $\kappa \mathrm{B}$ signaling system has been activated and that signaling peaked after $15 \mathrm{~min}$ reperfusion, indicating that the $\mathrm{NF}-\kappa \mathrm{B}$ signaling system may be very close to the release of inflammation triggered by renal epithelial cell damage signaling system (30). 
The results from the present study indicated that treatment with ursolic acid significantly reduced the increasing NF- $\kappa \mathrm{B}$ and caspase-3 level in the I/R-IAKI rat. Ma et al (28) suggested that ursolic acid protects against oxidative DNA damage and inflammation by inhibiting the activities of STAT3 and NF- $\mathrm{BB}$. Lu et al (31) suggested that ursolic acid improves cognitive impairments via NF- $\mathrm{NB}$-mediated inflammatory pathways in mice. By contrast, the present study found that NF- $\mathrm{BB}-$ mediated inflammatory pathways may be an important mechanims by which ursolic acid protects against I/R-IAKI.

In conclusion, the present study demonstrated that the renoprotective effects of ursolic acid on I/R-IAKI occur via oxidative stress, inflammation and inhibition of the activities of STAT3 and NF- $\kappa$ B. Ursolic acid may serve a potentially important role in the treatment of I/R-IAKI.

\section{References}

1. Kirkendall ES, Spires WL, Mottes TA, Schaffzin JK, Barclay C and Goldstein SL: Development and performance of electronic acute kidney injury triggers to identify pediatric patients at risk for nephrotoxic medication-associated harm. Appl Clin Inform 5 313-333, 2014.

2. Renhua L, Miaolin C, Junlin W, Qingwei W, Xiaoping X, Huili D, Weiming Z, Zhaohui N, Jiaqi Q and Yan Y: The level of the biomarkers at the time of nephrology consultation might predict the prognosis of acute kidney injury in hospitalized patients. Blood Purif 38: 89-95, 2014.

3. Li W, Qian J, Liu X, Zhang Q, Wang L, Chen D and Lin Z: Management of severe crush injury in a front-line tent ICU after 2008 Wenchuan earthquake in China: An experience with 32 cases. Crit Care 13: R178, 2009.

4. Tsai TT, Patel UD, Chang TI, Kennedy KF, Masoudi FA, Matheny ME, Kosiborod M, Amin AP, Messenger JC, Rumsfeld JS and Spertus JA: Contemporary incidence, predictors and outcomes of acute kidney injury in patients undergoing percutaneous coronary interventions: Insights from the NCDR Cath-PCI registry. JACC Cardiovasc Interv 7: 1-9, 2014

5. Zhou C, Wang R, Ding Y, Du L, Hou C, Lu D, Hao L and Lv W: Prognostic factors for acute kidney injury following transarteria chemoembolization in patients with hepatocellular carcinoma Int J Clin Exp Pathol 7: 2579-2586, 2014.

6. Chiang WC, Chien CT, Lin WW, Lin SL, Chen YM, Lai CF, $\mathrm{Wu} \mathrm{KD}$, Chao J and Tsai TJ: Early activation of bradykinin $\mathrm{B} 2$ receptor aggravates reactive oxygen species generation and renal damage in ischemia/reperfusion injury. Free Radic Biol Med 41: 1304-1314, 2006.

7. Du Y, Hou L, Guo J, Sun T, Wang X and Wu Y: Renal neutrophil gelatinase-associated lipocalin and kidney injury molecule-1 expression in children with acute kidney injury and Henoch-Schönlein purpura nephritis. Exp Ther Med 7: 1130-1134, 2014

8. Wang Z, Ge Y, Bao H, Dworkin L, Peng A and Gong R: Redox-sensitive glycogen synthase kinase $3 \beta$-directed control of mitochondrial permeability transition: Rheostatic regulation of acute kidney injury. Free Radic Biol Med 65: 849-858, 2013.

9. Sung FL, Zhu TY, Au-Yeung KK, Siow YL and O K: Enhanced MCP-1 expression during ischemia/reperfusion injury is mediated by oxidative stress and NF-kappaB. Kidney Int 62: $1160-1170,2002$.

10. Zhou Y, Li JS, Zhang X, Wu YJ, Huang K and Zheng L: Ursolic acid inhibits early lesions of diabetic nephropathy. Int J Mol Med 26: 565-570, 2010.

11. Gong YY, Liu YY, Yu S, Zhu XN, Cao XP and Xiao HP: Ursolic acid suppresses growth and adrenocorticotrophic hormone secretion in AtT20 cells as a potential agent targeting adrenocorticotrophic hormone-producing pituitary adenoma. Mol Med Rep 9: 2533-2539, 2014.

12. Novotný L, Vachálková A and Biggs D: Ursolic acid: An anti-tumorigenic and chemopreventive activity. Minireview. Neoplasma 48: 241-246, 2001
13. Hu Z, Gu Z, Sun M, Zhang K, Gao P, Yang Q and Yuan Y: Ursolic acid improves survival and attenuates lung injury in septic rats induced by cecal ligation and puncture. J Surg Res 194: 528-536, 2015.

14. Lien YH, Lai LW and Silva AL: Pathogenesis of renal ischemia/reperfusion injury: Lessons from knockout mice. Life Sci 74: 543-552, 2003.

15. Xia Y, Rao J, Yao A, Zhang F, Li G, Wang X and Lu L: Lithium exacerbates hepatic ischemia/reperfusion injury by inhibiting GSK- $3 \beta / N F-\kappa B$-mediated protective signaling in mice. Eur J Pharmacol 697: 117-125, 2012.

16. Li L, Zhang X, Cui L, Wang L, Liu H, Ji H and Du Y: Ursolic acid promotes the neuroprotection by activating Nrf2 pathway after cerebral ischemia in mice. Brain Res 1497: 32-39, 2013.

17. Tundis R, Nadjafi F and Menichini F: Angiotensin-converting enzyme inhibitory activity and antioxidant properties of Nepeta crassifolia Boiss \& Buhse and Nepeta binaludensis Jamzad. Phytother Res 27: 572-580, 2013.

18. Hagiwara M, Shen B, Chao L and Chao J: Kallikrein-modified mesenchymal stem cell implantation provides enhanced protection against acute ischemic kidney injury by inhibiting apoptosis and inflammation. Hum Gene Ther 19: 807-819, 2008.

19. Senthil S, Chandramohan G and Pugalendi KV: Isomers (oleanolic and ursolic acids) differ in their protective effect against isoproterenol-induced myocardial ischemia in rats. Int J Cardiol 119: 131-133, 2007.

20. Tawfik MK: Renoprotective activity of telmisartan versus pioglitazone on ischemia/reperfusion induced renal damage in diabetic rats. Eur Rev Med Pharmacol Sci 16: 600-609, 2012.

21. Jin X, Zhang Y, Li X, Zhang J and Xu D: C-type natriuretic peptide ameliorates ischemia/reperfusion-induced acute kidney injury by inhibiting apoptosis and oxidative stress in rats. Life Sci 117: 40-45, 2014.

22. Ma JQ, Ding J, Zhang L and Liu CM: Ursolic acid protects mouse liver against $\mathrm{CCl} 4$-induced oxidative stress and inflammation

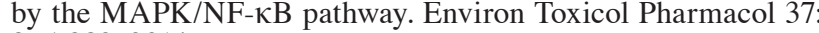
975-983, 2014.

23. Ramachandran S and Prasad NR: Effect of ursolic acid, a triterpenoid antioxidant, on ultraviolet-B radiation-induced cytotoxicity, lipid peroxidation and DNA damage in human lymphocytes. Chem Biol Interact 176: 99-107, 2008.

24. Wang T, Yuan W, Liu Y, Zhang Y, Wang Z, Zhou X, Ning G, Zhang L, Yao L, Feng S and Kong X: The role of the JAK-STAT pathway in neural stem cells, neural progenitor cells and reactive astrocytes after spinal cord injury. Biomed Rep 3: 141-146, 2015.

25. Hsieh HL and Yang CM: Role of redox signaling in neuroinflammation and neurodegenerative diseases. Biomed Res Int 2013: 484613, 2013.

26. Susnik N, Sörensen-Zender I, Rong S, von Vietinghoff S, Lu X, Rubera I, Tauc M, Falk CS, Alexander WS, Melk A, et al: Ablation of proximal tubular suppressor of cytokine signaling 3 enhances tubular cell cycling and modifies macrophage phenotype during acute kidney injury. Kidney Int 85: 1357-1368, 2014.

27. Pathak AK, Bhutani M, Nair AS, Ahn KS, Chakraborty A, Kadara H, Guha S, Sethi G and Aggarwal BB: Ursolic acid inhibits STAT3 activation pathway leading to suppression of proliferation and chemosensitization of human multiple myeloma cells. Mol Cancer Res 5: 943-955, 2007.

28. Ma JQ, Ding J, Xiao ZH and Liu CM: Ursolic acid ameliorates carbon tetrachloride-induced oxidative DNA damage and inflammation in mouse kidney by inhibiting the STAT3 and NF- $\mathrm{BB}$ activities. Int Immunopharmacol 21: 389-395, 2014.

29. Chang KK, Liu LB, Li H, Mei J, Shao J, Xie F, Li MQ and Li DJ: TSLP induced by estrogen stimulates secretion of MCP-1 and IL-8 and growth of human endometrial stromal cells through JNK and NF- $\kappa \mathrm{B}$ signal pathways. Int J Clin Exp Pathol 7: 1889-1899, 2014.

30. Cao CC, Ding XQ, Ou ZL, Liu CF, Li P, Wang L and Zhu CF: In vivo transfection of NF-kappaB decoy oligodeoxynucleotides attenuate renal ischemia/reperfusion injury in rats. Kidney Int 65: 834-845, 2004

31. Lu J, Wu DM, Zheng YL, Hu B, Cheng W, Zhang ZF and Shan Q: Ursolic acid improves high fat diet-induced cognitive impairments by blocking endoplasmic reticulum stress and $\mathrm{I}_{\kappa} \mathrm{B}$ kinase $\beta /$ nuclear factor- $\mathrm{\kappa} B$-mediated inflammatory pathways in mice. Brain Behav Immun 25: 1658-1667, 2011. 